\title{
Revista Colombiana de

\section{Evaluación de la respuesta a la terapia de resincronización cardíaca en un grupo de pacientes que asisten a un programa de falla cardíaca}

\section{Catalina Gallego Muñoz*, Clara Inés Saldarriaga, Eliana Mabel Cañas Arenas, Carolina Delgado Gómez y Carolina González Berrío}

\author{
Departamento de Cardiología Clínica, Clínica CardioVID, Medellín, Colombia
}

Recibido el 2 de febrero de 2017; aceptado el 10 de abril de 2017

Disponible en Internet el 8 de julio de 2017

\author{
PALABRAS CLAVE \\ Insuficiencia \\ cardíaca; \\ Terapia de \\ resincronización \\ cardíaca; \\ Resultado del \\ tratamiento
}

\begin{abstract}
Resumen
Introducción: la terapia de resincronización cardiaca es una de las estrategias de tratamiento actuales para pacientes con insuficiencia cardiaca y función sistólica deprimida, que ha demostrado producir un alivio significativo en los síntomas, así como mejoría en la función ventricular izquierda y en la supervivencia a largo plazo.

Objetivo: evaluar la respuesta a la terapia de resincronización cardíaca después de seis meses de seguimiento.

Metodología: estudio observacional analítico de cohorte retrospectivo, con análisis anidado de casos y controles, en el que se incluyeron pacientes mayores de 18 años pertenecientes a una clínica de falla cardíaca y que fueran portadores de un dispositivo de resincronización cardíaca. Se realizó análisis univariado de las características clínicas y ecocardiográficas y posteriormente un análisis bivariado a las variables relacionadas con la respuesta a la terapia.

Resultados: se incluyeron 92 pacientes con historia de falla cardíaca e indicación de terapia de resincronización; el $32,6 \%$ de los pacientes tenían cardiopatía isquémica y el $67,4 \%$ no isquémica, con una fracción de eyección promedio de 18,9\%. Durante el seguimiento a los 6 primeros meses no se presentó ningún caso de muerte ni de trasplante cardíaco. Se definieron como respondedores el $47,8 \%$ de los pacientes, en tanto que el $52,2 \%$ restante fue clasificado como no respondedores. El porcentaje de pacientes que no requirieron hospitalizaciones luego de la terapia fue del $51,1 \%$, y el $65,2 \%$ mejoró la clase funcional y $68,5 \%$ la fracción de eyección.
\end{abstract}

\footnotetext{
* Autor para correspondencia.

Correo electrónico: catagamu@hotmail.com (C. Gallego Muñoz).
} 
Conclusión: en el $47,8 \%$ de la población estudiada se observó una respuesta a la terapia de resincronización cardiaca adecuada evaluada de forma combinada con parámetros clínicos y ecocardiográficos, hecho que se tradujo en una mejoría relevante en términos de fracción de eyección y clase funcional, además de menor número de hospitalizaciones por falla cardíaca, al igual que ausencia de requerimiento de trasplante cardíaco y de muertes por causa cardiovascular.

(c) 2017 Sociedad Colombiana de Cardiología y Cirugía Cardiovascular. Publicado por Elsevier España, S.L.U. Este es un artículo Open Access bajo la licencia CC BY-NC-ND (http:// creativecommons.org/licenses/by-nc-nd/4.0/).

\section{KEYWORDS}

Heart failure;

Cardiac

synchronisation

therapy;

Treatment results

\section{Introducción}

La falla cardíaca es una enfermedad de alta prevalencia y mortalidad. La terapia de resincronización cardíaca ha cambiado el tratamiento de los pacientes con insuficiencia cardíaca refractaria al manejo farmacológico, dado que se ha demostrado que produce alivio significativo de los síntomas al tiempo que mejora la función ventricular izquierda y la supervivencia a largo plazo ${ }^{1}$.

Está indicada en los pacientes con disincronía definida como QRS con una duración mayor a $120 \mathrm{~ms}$ y bloqueo de rama izquierda del haz de His, en un tratamiento farmacológico óptimo de falla cardíaca por lo menos por tres meses y que pese a ello persistan en clase funcional ॥ de la clasificación de la NYHA (New York Heart Association) o mayor ${ }^{2}$.

El entusiasmo por la sorprendente eficacia de la terapia de resincronización en pacientes con falla cardíaca y alteración de la conducción ventricular se ha visto aminorado por la observación de que una proporción variable de pacientes elegibles parece no beneficiarse de este tratamiento, o son "no-respondedores" 3 . El espectro de respuesta a la terapia de resincronización cardiaca varía desde el franco deterioro, a ninguna mejoría apreciable o a simplemente una mejoría mínima ${ }^{4}$. Se estima que aproximadamente el $30-40 \%$ de los pacientes que reciben terapia de resincronización cardiaca no responden al tratamiento. En los estudios que utilizan definiciones subjetivas como la valoración de la clase funcional, las tasas de no respuesta son mucho menores, y varían desde el $10 \%$ hasta el $26 \%$. En aquellos estudios donde se evalúan parámetros objetivos, como el remodelamiento del ventrículo izquierdo, la no respuesta puede ser tan alta como hasta del $40-46 \% 5$.

Las razones por las cuales algunos pacientes no responden a la terapia de resincronización son múltiples, entre estas: selección inadecuada, posición subóptima de los 
electrodos y programación inadecuada del dispositivo $0^{6,7}$. Algunos autores proponen que en las formas más avanzadas de la falla cardíaca, en donde existe una disfunción ventricular severa e irreversible, es menos probable que exista una respuesta apropiada a la resincronización ${ }^{8,9}$. Es por esto que en la literatura actual existe un interés creciente por definir las características de la población no respondedora para establecer estrategias adicionales de manejo y mejorar los criterios de selección de los pacientes candidatos a dicha terapia ${ }^{10,11}$.

A la fecha es poco lo que se conoce dentro de nuestra población, acerca de la frecuencia de respondedores a la terapia, características clínicas, ecocardiográficas u otras variables que pudiesen tener impacto en el manejo subsecuente de este grupo de pacientes. Es de gran importancia conocer cómo se comportan los pacientes de la práctica clínica diaria con sus múltiples comorbilidades, enfermedades avanzadas y además con implante de dispositivos en sitios con menor experiencia que aquellos donde se reclutan pacientes en los ensayos clínicos controlados. En este estudio se describe la respuesta a la terapia de resincronización cardiaca, caracterizando un grupo de pacientes que se encuentran en seguimiento en una clínica de falla cardíaca.

\section{Métodos}

Estudio observacional analítico de cohorte, retrospectivo, con análisis anidado de casos y controles, en el que se consideraron como casos, aquellos pacientes pertenecientes al programa de falla cardíaca que respondieron a la terapia de resincronización cardiaca, definiendo respuesta como la mejoría en una (I) clase funcional de la clasificación de NYHA, o la reducción en el diámetro del fin de sístole mayor o igual al 15\%, o un incremento absoluto en la fracción de eyección del ventrículo izquierdo mayor a $5 \%$, y que estuvieran libres de hospitalizaciones por descompensación aguda de falla cardíaca o muerte por causa cardiovascular en un período de seguimiento de seis meses. Se definieron como controles, aquellos pacientes que no respondieron a la terapia de resincronización, según los criterios ya definidos. El estudio fue aprobado por el Comité de Ética de Investigación en Salud de la institución.

\section{Población}

Se incluyeron todos aquellos pacientes mayores de 18 años, portadores de dispositivo de resincronización (resincronizador o resincronizador-desfibrilador) que estuvieran inscritos en un programa de falla cardíaca entre junio de 2012 y junio de 2014. Se consideraron criterios de exclusión la ausencia de seguimiento clínico o ecocardiográfico a los seis meses posteriores al implante del resincronizador y la no disponibilidad de los datos clínicos completos que corresponden a las variables de estudio, en la historia clínica electrónica/física. Se realizó un muestreo por conveniencia donde se incluyeron todos los pacientes que cumplieran con los criterios de elegibilidad en el periodo de estudio.

\section{Procedimientos de estudio}

Se evaluó la historia clínica de los pacientes que se encontraban vinculados al programa de falla cardíaca y que fueran portadores de un dispositivo de resincronización cardíaca, tomando como punto cero el momento en el cual se implantó el dispositivo y como punto de seguimiento la primera evaluación posterior al implante (para la evaluación ecocardiográfica se tomó como punto cero la ecocardiografía realizada al momento del implante del dispositivo y la de seguimiento corresponde a aquella efectuada entre el tercer al sexto mes posterior al implante). La estimación de la fracción de eyección del ventrículo izquierdo se calculó a través del método de Simpson mientras que la medición del diámetro de fin de sístole del ventrículo izquierdo se realizó al final de la sístole ventricular adquiriendo la imagen en una ventana paraesternal eje largo. Todas las mediciones se hicieron con imágenes de tiempo real.

Se usó una hoja prediseñada para la recolección de datos y una base de datos para el estudio en Microsoft Excel 2010 con validación de datos acorde con el tipo de variables para disminuir los riesgos de errores a la hora de ingresar los datos.

\section{Análisis estadístico}

La descripción de las características basales de tipo demográficas, clínicas y ecocardiográficas, se hizo mediante media o mediana, como medida de tendencia central, y desviación estándar o rango intercuartil, como medida de dispersión, según la distribución, en el caso de las variables cuantitativas. Las variables cualitativas se describieron mediante frecuencias absolutas y relativas. Se realizó un análisis univariado con todas las características clínicas y ecocardiográficas de los pacientes, usando el test T de Student y la prueba de Chi cuadrado según el tipo de variable y la distribución, considerando como estadísticamente significativos los valores de $p$ menores de 0,05 . Para las variables que no tenían distribución normal, se usó la prueba estadística de Mann-Whitney. Se llevó a cabo un análisis bivariado de las variables que habían reportado en otros estudios como factores modificadores de la respuesta a la terapia de resincronización cardiaca ${ }^{12-16}$. Se utilizó la medida de razón de disparidad (OR, su sigla en inglés) con sus respectivos intervalos de confianza del $95 \%$ y se calcularon sus valores de $p$ aplicando la prueba de Chi cuadrado; se consideraron estadísticamente significativos los valores de $p$ menores de 0,05. Para todos los análisis se usó el programa SPSS versión 20.

\section{Resultados}

Se incluyeron en total 92 pacientes con historia de insuficiencia cardíaca e indicación de terapia de resincronización, de los cuales el $58,7 \%$ eran hombres y el $41,3 \%$ mujeres, con un promedio de edad de 64,6 años. El 32,6\% tenían cardiopatía isquémica y el $67,4 \%$ etiología no isquémica; la fracción de eyección promedio al ingreso fue $18,9 \%$. El $91,3 \%$ tenían, en el electrocardiograma de superficie previo al implante del dispositivo, morfología de bloqueo de rama izquierda y una duración promedio del QRS de 151,9 milisegundos (ms). Las principales comorbilidades descritas 
Tabla 1 Características basales de la población

\begin{tabular}{|c|c|}
\hline \multicolumn{2}{|l|}{ Característica } \\
\hline Edad, años & $65(57-73)^{*}$ \\
\hline Género, masculino \% & 58,7 \\
\hline Etiología isquémica \% & 32,6 \\
\hline QRS duración, milisegundos & $\begin{array}{l}151,9(\mathrm{DE} \\
21,0)^{*}\end{array}$ \\
\hline \multicolumn{2}{|l|}{ QRS morfología } \\
\hline $\mathrm{BRIHH}^{\dagger} \%$ & 91,3 \\
\hline $\mathrm{BRDHH}^{\ddagger} \%$ & 2,2 \\
\hline $\begin{array}{l}\text { Trastorno inespecífico de la conducción } \\
\text { intraventricular \% }\end{array}$ & 5,4 \\
\hline Diabetes \% & 23,9 \\
\hline Hipertensión arterial \% & 68,5 \\
\hline Fibrilación auricular \% & 31,5 \\
\hline \multicolumn{2}{|l|}{ Clase funcional NYHA } \\
\hline NYHA ॥ \% & 32,6 \\
\hline NYHA III \% & 54,3 \\
\hline NYHA Iv \% & 13 \\
\hline $\begin{array}{l}\text { Fracción de eyección del ventrículo } \\
\text { izquierdo, porcentaje }\end{array}$ & $\begin{array}{l}18,9(\mathrm{DE} \\
7,2)^{*}\end{array}$ \\
\hline $\begin{array}{l}\text { Diámetro de fin de sístole del ventrículo } \\
\text { izquierdo, milímetros }\end{array}$ & $5,5(\mathrm{DE} 1,0)^{*}$ \\
\hline \multicolumn{2}{|c|}{ Localización del electrodo del ventrículo izquierdo } \\
\hline Anterior/anterolateral \% & 30,4 \\
\hline Lateral \% & 2,2 \\
\hline Posterior/posterolateral \% & 47,9 \\
\hline Epicárdico \% & 22,8 \\
\hline \multicolumn{2}{|l|}{ Medicamentos } \\
\hline Betabloqueadores \% & 91,3 \\
\hline IECA/ARAII"*\% & 84,7 \\
\hline Espironolactona \% & 76 \\
\hline Diurético de asa \% & 79,3 \\
\hline Digoxina \% & 76 \\
\hline
\end{tabular}

BRIHH: bloqueo rama izquierda del haz de His. BRDHH: bloqueo rama derecha del haz de His. NYHA: New York Heart Association. IECA: inhibidores de la enzima convertidora de angiotensina/ARAll: antagonistas de los receptores de la angiotensina ॥.

" La edad se muestra en mediana y rango intercuartil; los demás datos en media y desviación estándar (DE).

dentro de la población fueron la hipertensión arterial en el $68,5 \%$, fibrilación auricular en el $31,5 \%$ y diabetes mellitus tipo 2 en el $23,9 \%$. En la tabla 1 se presentan las características basales de los pacientes incluidos.

Se definieron como respondedores, según lo establecido para el estudio (desenlace combinando de criterios clínicos y ecocardiográficos), el $47,8 \%$ de los pacientes, que fueron aquellos que no fueron hospitalizados ni trasplantados en los seis meses de seguimiento y además tuvieron mejoría en clase funcional o en fracción de eyección o disminución del diámetro de fin de sístole. El 52,2\% de la población restante se clasificó como no respondedora. En la tabla 2 se reportan la respuesta a la terapia de resincronización según los diferentes parámetros evaluados y la respuesta con base en la definición combinada.
Tabla 2 Respuesta a la terapia de resincronización

\begin{tabular}{lc}
\hline Característica clínica y/o ecocardiográfica & $\%(\mathrm{~N})$ \\
\hline Respuesta a la terapia de resincronización & $47,8(44)$ \\
No respuesta a la terapia de resincronización & $52,2(48)$ \\
Mortalidad a 6 meses & $0(94)$ \\
Hospitalizaciones por falla cardíaca descompensada & $51,1(47)$ \\
$\quad$ Ninguna & $48,9(45)$ \\
1 ó más hospitalizaciones & \\
Clase funcional & $65,2(60)$ \\
$\quad$ Mejoría en al menos I clase NYHA* & $34,8(32)$ \\
$\quad$ Sin mejoría en al menos I clase NYHA* & \\
Fracción de eyección del ventrículo izquierdo & $68,5(63)$ \\
$\quad$ Mejoría en al menos 5\% & $31,5(29)$ \\
$\quad$ No mejoría en al menos 5\% & $23,9(22)$ \\
Diámetro de fin de sístole del ventrículo izquierdo & \\
$\quad$ Disminución mayor al 15\% & $37(34)$ \\
$\quad$ Disminución inferior al 15\% &
\end{tabular}

La clase funcional de la NYHA en el seguimiento se distribuyó de la siguiente manera: clase । $20,7 \%$, clase ॥ $55,4 \%$, clase III 17,4\% y clase Iv 6,5\%. En el seguimiento a 6 meses, la fracción de eyección promedio fue de 29,1\% (DE 13,6), siendo del $54,5 \%$ en el grupo considerado como respondedor y del $39 \%$ en los pacientes no respondedores $(p=0,005)$. El promedio del diámetro de fin de sístole del ventrículo izquierdo para los pacientes que tenían el dato disponible fue 4,9 mm (DE 1,3), siendo en promedio 4,7 mm (DE 1,2) en aquellos que se clasificaron como respondedores y de $5,1 \mathrm{~mm}$ $(D E 1,4)$ en los no respondedores $(p=0,222)$.

La discriminación de las variables según la respuesta a la terapia se muestra en la tabla 3 . Se reportan los OR para el análisis bivariado de los factores descritos en la literatura que favorecen una adecuada respuesta a la terapia de resincronización cardiaca ${ }^{12-16}$, sin encontrar diferencias estadísticamente significativas.

\section{Discusión}

Los resultados de este estudio muestran cómo los criterios para la selección e implante de un dispositivo de terapia de resincronización cardiaca en un grupo de 92 pacientes pertenecientes a una clínica de falla cardíaca, son aquellos que la literatura y, específicamente las guías actuales de manejo de insuficiencia cardíaca, han determinado como los criterios de indicación de terapia de resincronización ${ }^{2}$.

Todos los pacientes que recibieron la terapia tenían previo al implante una fracción de eyección menor del $35 \%$, la mayoría estaban clasificados al ingreso como clases funcionales entre $॥$ y III de la NYHA. En cuanto al electrocardiograma, el promedio del QRS previo al implante del dispositivo fue de 151,9 milisegundos y la mayoría tenían morfología de bloqueo de rama izquierda. Al evaluar la terapia farmacológica se evidenció que la mayoría recibían, al momento de inclusión en el estudio, los principales grupos farmacológicos de la terapia óptima de falla cardíaca, como son los betabloqueadores y los inhibidores de la enzima 
Tabla 3 Respondedores vs. no respondedores

\begin{tabular}{|c|c|c|c|c|c|}
\hline Variable & Respondedor (n) & No respondedor $(n)$ & $p$ & OR & IC \\
\hline Sexo, femenino\% & $38,6(17)$ & $43,8(21)$ & 0,619 & 0,8 & $0,352-1,862$ \\
\hline Etiología no isquémica \% & $72,7(32)$ & $27,3(12)$ & 0,296 & 1,6 & $0,661-3,873$ \\
\hline \multicolumn{6}{|l|}{ QRS duración } \\
\hline$>150$ milisegundos & $33,6(31)$ & $34,7(32)$ & 0,751 & 1,15 & $0,47-2,79$ \\
\hline$<150$ milisegundos & $14,1(13)$ & $17,3(16)$ & & & \\
\hline \multicolumn{6}{|l|}{ QRS morfología } \\
\hline $\mathrm{BRIHH}^{*}$ & $44,5(41)$ & $46,7(43)$ & 0,762 & 1,27 & $0,268-6,031$ \\
\hline Otra morfología diferente & $4,3(4)$ & $4,3(4)$ & & & \\
\hline Fibrilación auricular & $22,7(10)$ & $77,3(34)$ & 0,082 & 0,449 & $0,180-1,118$ \\
\hline \multicolumn{6}{|l|}{ Posición electrodo } \\
\hline Localización no epicárdica & $39,1(36)$ & $38(35)$ & 0,310 & 1,671 & $0,617-4,526$ \\
\hline Localización epicárdica & $8,6(8)$ & $14,1(13)$ & & & \\
\hline
\end{tabular}

BRIHH: bloqueo rama izquierda del haz de His. NYHA: New York Heart Association.

convertidora de angiotensina (IECA) o antagonistas del receptor tipo II de la aldosterona, en caso de intolerancia a los IECA, y un alto porcentaje recibía diuréticos.

En cuanto a la causa de la falla cardíaca, la mayoría tenía falla de etiología no isquémica, siendo la cardiopatía isquémica la causa de la insuficiencia cardiaca en cerca del $32 \%$ de los pacientes. Estos datos son cercanos a los reportados en una publicación reciente, en la que se incluyeron pacientes pertenecientes a las clínicas de falla cardíaca del país con una muestra de 4.239 casos, mostrándose etiología isquémica en el $38,7 \%$, idiopática en el $21,6 \%$, hipertensiva en el $12,2 \%$, valvular en el $10,5 \%$ y chagásica en el $10,3 \%{ }^{17}$. Se resalta la importancia que tiene dentro de nuestra población la patología no isquémica, sobre todo la enfermedad de Chagas y la enfermedad valvular de origen reumático. En Colombia, el $18 \%$ de la población vive en áreas endémicas de la enfermedad de Chagas y se estima que 1,3 millones de personas están infectadas ${ }^{18}$. Es por esto que posiblemente se requiera en el futuro más investigación para conocer con mayor precisión la etiología de la falla dentro de nuestra población a través de estudios poblacionales o registros más amplios, para establecer con precisión la etiología y el manejo de la misma.

Las comorbilidades más descritas en este estudio fueron la hipertensión arterial y la diabetes mellitus tipo 2, similar a lo que se describe en la literatura ${ }^{19}$; además, hay un número importante de pacientes con fibrilación auricular cercano al $32 \%$ que podría relacionarse con respuesta inadecuada a la terapia de resincronización, en el caso de tener menor porcentaje de estimulación biventricular por ritmo de fibrilación auricular permanente 20,21 .

Respecto a la respuesta a la terapia de resincronización cardiaca en este grupo de pacientes de la práctica clínica diaria, se encontró un porcentaje de respuesta a la terapia cercano al $48 \%$, según lo definido por el estudio, que es un compuesto de desenlaces clínicos y ecocardiográficos. Una publicación reciente ${ }^{22}$, en la que evalúa la respuesta a la terapia de resincronización cardiaca en términos de desenlaces combinados, clínicos y ecocardiográficos, demostró una tasa de respuesta a dicha terapia, en promedio del $40-50 \%$, similar a lo que se reporta en nuestro estudio.
Se evidencia además en nuestra población que, desde el punto de vista de desenlaces clínicos, la respuesta en parámetros individuales fue similar a lo reportado en la literatura, en donde los porcentajes varían desde valores tan amplios como del $50-70 \%$ cuando se utilizan parámetros como hospitalizaciones o mortalidad; en desenlaces como mejoría de la clase funcional la tasas de respuesta promedio son cercanas al $80 \%{ }^{11}$. En nuestra población no se reportaron muertes por causa cardiovascular ni requerimiento de trasplante cardiaco en los seis primeros meses de seguimiento. Se observó que el $51,1 \%$ de la población no tuvo hospitalizaciones por falla cardíaca descompensada, además, el $65,2 \%$ mejoró su clase funcional. Estos parámetros clínicos son probablemente los más relevantes y los que expliquen los grandes costos de la falla cardíaca en el mundo.

En cuanto a los desenlaces ecocardiográficos, se ha descrito que la disminución en el diámetro de fin de sístole del ventrículo izquierdo mayor o igual al $15 \%$ o el incremento en el valor absoluto en la fracción de eyección mayor al $5 \%{ }^{5}$, evalúan la reversión en el remodelamiento cardíaco del ventrículo izquierdo luego de tres a seis meses de terapia, y constituyen los parámetros ecocardiográficos con mejor valor pronóstico para los desenlaces clínicos $^{22}$. Cuando se evalúan estos parámetros ecocardiográficos, la respuesta a la terapia de resincronización cardiaca puede ser tan variable como del $30-60 \%{ }^{11}$. En los pacientes incluidos en este estudio, la reversión en la remodelación medida por la disminución en el diámetro de fin de sístole fue el parámetro que presentó menos cambio, aunque no pudo evaluarse en todos los pacientes puesto que no estaba registrado en las ecocardiografías al ingreso o al seguimiento. No fue posible obtener volúmenes ventriculares con su respectiva indexación dado que no estaban medidos en las ecocardiografías tanto al ingreso como en el seguimiento. En cuanto a la fracción de eyección, se evidenció un incremento de al menos $5 \%$ en el $68,5 \%$ de los casos, con un aumento mayor en aquellos que se consideraron respondedores comparados con los no-respondedores (promedio de $54,5 \%$ vs. $39 \%$ respectivamente, $p=0,005)$. La medición de esta variable estuvo ampliamente disponible en los datos analizados, dado que es uno de los parámetros al que más seguimiento se le hace 
y que se mide de forma rutinaria en las ecocardiografías de los pacientes con falla cardíaca.

En resumen, la evaluación de la respuesta a la terapia de resincronización cardiaca es aun controversial dada la falta de consenso por parte de la literatura para definir los parámetros de respuesta y tiempo esperado para la misma, además existen múltiples definiciones que en la mayoría de los estudios corresponden a desenlaces duros como muerte por causa cardiovascular y hospitalización por empeoramiento de falla cardíaca, mientras que otros autores pretenden definir la respuesta a la terapia de resincronización a través de desenlaces compuestos que involucren variables clínicas y resultados objetivos desde el punto de vista funcional (test de caminata de seis minutos) o ecocardiográfico ${ }^{1,3,5}$. Como se había descrito previamente son pocos los datos que se conocen en nuestra población sobre la respuesta a la terapia de resincronización. Franco et al., en el año 2014 publicaron su experiencia en 70 pacientes en los que se implantó un dispositivo de resincronización o un desfibrilador, aunque se concluyó que las características de estos pacientes eran similares a los de las publicaciones mundiales, tanto para la etiología de la falla cardíaca, como para el perfil de comorbilidades y el manejo farmacológico. No se evaluó la respuesta a la terapia, por tanto se requieren más estudios con diseños metodológicos más estrictos dentro de la población colombiana para conocer con certeza como se comporta la respuesta a la terapia de resincronización cardiaca.

En lo concerniente a los factores que se consideraron en el análisis bivariado como posibles determinantes de la respuesta favorable a la terapia de resincronización cardiaca, ninguno de los resultados obtenidos tuvo precisión ni significancia estadística. Condiciones como el sexo femenino, que se ha sido descrito en la literatura como un factor predictor de buena respuesta a la terapia de resincronización con mejoría en la supervivencia a largo plazo ${ }^{23}$, no demostró en nuestra población ninguna diferencia. Así mismo, la morfología de bloqueo de rama izquierda al momento del implante que estaba presente en la mayoría de los pacientes de este estudio ${ }^{24}$, y el tamaño del complejo QRS que fue en promedio superior a $150 \mathrm{~ms}$, también han sido reportados en la literatura como predictores de buena respuesta ${ }^{6}$, pero en esta población no se pudo establecer ninguna diferencia significativa. Otros factores estudiados como la etiología de la falla cardíaca, han demostrado que el origen isquémico tiene menor probabilidad de responder a la resincronización pues la extensión de la cicatriz miocárdica es un determinante de la falta de respuesta ${ }^{11}$; en nuestra población tampoco pudo demostrarse que fuera un factor determinante de la respuesta. Por último, la localización óptima del electrodo del ventrículo izquierdo es crucial para obtener el mayor beneficio de la terapia de resincronización. La evidencia sugiere que las localizaciones posterior y lateral son las que mayor beneficio brindan desde el punto de vista hemodinámico, mientras que la posición anterior o la inserción epicárdica es un factor que aumenta el riesgo de no respuesta de dos a tres veces ${ }^{25}$. En este estudio no pudo demostrarse que la localización del electrodo del ventrículo izquierdo se comportara como un factor que se relacione con una mejor respuesta a la terapia. Todo lo expuesto parece obedecer al tamaño de la muestra de esta publicación, de modo que se requieren más estudios para conocer en nuestra población cuales son los factores que influyen en la ausencia de respuesta a la terapia.

La principal limitación de este estudio fue el tamaño de la muestra. No fue posible demostrar de forma estadísticamente significativa que los factores que clásicamente se han descrito como relacionados con mejor respuesta a la terapia de resincronización cardiaca, influyeran en esta población. Así mismo, la naturaleza retrospectiva del estudio y la duración del seguimiento pueden afectar la respuesta a muchas de las variables evaluadas. Otra limitante del estudio fue la valoración incompleta desde el punto de vista ecocardiográfico, como se anotó previamente el diámetro de fin de sístole no estaba disponible en todos los pacientes incluidos y tampoco se pudo evaluar la respuesta en términos de volúmenes ventriculares con su respectiva indexación por ausencia de estos datos en las ecocardiografías tomadas a los pacientes.

A pesar de estas limitaciones, dado que en Colombia no se conocen muchos estudios sobre respuesta a la resincronización cardíaca en el mundo real, es valioso el análisis de estos resultados y sobre todo saber cómo se comportan los pacientes de la práctica clínica diaria, con sus múltiples comorbilidades, enfermedades avanzadas, con implante de dispositivos en sitios con menor experiencia que aquellos donde se reclutan los pacientes en los ensayos clínicos controlados.

\section{Conclusiones}

En síntesis, la evaluación de la respuesta a la terapia de resincronización es un tema aún controversial por la falta de consenso en la literatura. En este grupo de pacientes la respuesta a la terapia es cercana al $48 \%$, evaluando desenlaces compuestos clínicos y ecocardiográficos, y con mejoría en clase funcional y en la fracción de eyección superiores al 60\%. Los resultados del estudio son cercanos a los reportados en la literatura, principalmente por desenlaces clínicamente relevantes como la mejoría en la clase funcional y el número de hospitalizaciones, que son los que explican los grandes costos de la falla cardíaca.

\section{Responsabilidades éticas}

Protección de personas y animales. Los autores declaran que para esta investigación no se han realizado experimentos en seres humanos ni en animales.

Confidencialidad de los datos. Los autores declaran que han seguido los protocolos de su centro de trabajo sobre la publicación de datos de pacientes.

Derecho a la privacidad y consentimiento informado. Los autores declaran que en este artículo no aparecen datos de pacientes.

\section{Financiación}

Ninguna 


\section{Conflicto de interés}

\section{Ninguno}

\section{Bibliografía}

1. Linde C, Ellenbogen K, McAlister F. Cardiac resynchronization therapy (CRT): Clinical trials, guidelines, and target populations. Heart Rhythm. 2012;9:S3-13.

2. Brignole M, Auricchio A, Baron-Esquivias G, Bordachar P, Boriani G, Breithardt OA, et al., Developed in collaboration with the European Heart Rhythm Association (EHRA). ESC Guidelines on cardiac pacing and cardiac resynchronization therapy: the Task Force on cardiac pacing and resynchronization therapy of the European Society of Cardiology (ESC). Eur Heart J. 2013;34:2281-329.

3. Auricchio A, Prinzen F. Non-responders to cardiac resynchronization therapy-the magnitude of the problem and the issues. Circ J. 2011;75:521-7.

4. Tang WH, Boehmer J, Gras D. Multispecialty approach: The need for heart failure disease management for refining cardiac resynchronization therapy. Heart Rhythm. 2012;9:S45-50.

5. Shanks M, Delgado V, Ng AC, Mooyaart E, Bertini M, Marsan NA, et al. Clinical and echocardiographic predictors of nonresponse to cardiac resynchronization therapy. Am Heart J. 2011;161:552-7.

6. Bryant AR, Wilton SB, Lai MP, Exner DV. Association between QRS duration and outcome with cardiac resynchronization therapy: a systematic review and meta-analysis. J Electrocardiol. 2013;46:147-55

7. Adabag S, Roukoz H, Anand IS, Moss AJ. Cardiac resynchronization therapy in patients with minimal heart failure: a systematic review and meta-analysis. J Am Coll Cardiol. 2011;58:935-41.

8. Bordachar P, Eschalier R, Lumens J, Ploux S. Optimal strategies on avoiding CRT nonresponse. Curr Treat Options Cardio Med. 2014;16:299.

9. Tu R, Zhong G, Zeng Z, Wu W, Wu H, Cao X, et al. Cardiac resynchronization therapy in patients with mild heart failure: $a$ systematic review and meta-analysis of randomized controlled trials. Cardiovasc Drugs Ther. 2011;25:331-40.

10. Vidal B, Delgado V, Mont L, Poyatos S, Silva E, Angeles Castel $M$, et al. Decreased likelihood of response to cardiac resynchronization in patients with severe heart failure. Eur J Heart Fail. 2010;12:283-7.

11. Zhang $Q$, Zhou $Y$, Yu CM. Incidence, definition, diagnosis, and management of the cardiac resynchronization therapy nonresponder. Curr Opin Cardiol. 2015;30:40-9.

12. Upadhyay G, Choudrhy N, Auricchio A, Ruskin J, Sing J. Cardiac resynchronization patients with atrial fibrillation a meta analysis of prospective cohort studies. J Am Coll Cardiol. 2008;52:1239-46.

13. Healey J, Hohnloser SH, Exner DV, Birnie DH, Parkash R, Connolly SJ, et al. Cardiac Resynchronization therapy in patients with permanent atrial fibrillation Results From the Resynchronization for Ambulatory Heart Failure Trial (RAFT). Circ Heart Fail. 2012;5:566-70.

14. Arshad A, Moss AJ, Foster E, Padeletti L, Barsheshet A, Goldenberg I, et al. Cardiac resynchronization therapy is more effective in women than in men The MADIT-CRT (Multicenter Automatic Defibrillator Implantation Trial With Cardiac Resynchronization Therapy) Trial. J Am Coll Cardiol. 2011;57: 813-20.

15. Sipahi I, Carrigan T, Rowland D, Stamble BS, Fang J. Impact of QRS duration on clinical event reduction with cardiac resynchronization therapy Meta-analysis of Randomized Controlled Trials. Arch Intern Med. 2011;171:1454-62.

16. Spragg D, Dong J, Fetics BJ, Helm R, Marine JE, Cheng A, et al. Optimal left ventricular endocardial pacing sites for cardiac resynchronization therapy in patients with ischemic cardiomyopathy. J Am Coll Cardiol. 2010;56:774-81.

17. Gómez E. Introducción,;1; epidemiología de la falla cardiaca e historia de las clínicas de falla cardiaca en Colombia. Rev Colomb Cardiol. Publicado On Line. http://dx.doi.org/ 10.1016/j.rccar.2016.01.004.

18. Tamayo DC, Rodriguez VA, Rojas MX, Rincon M, Franco C, Ibarra MT, et al. Costos ambulatorios y hospitalarios de la falla cardiaca en dos hospitales de Bogotá. Acta Médica Colombiana. 2013;38.

19. Mentz RJ, Kelly JP, von Lueder TG, Voors AA, Lam CS, Cowie $M R$, et al. Noncardiac comorbidities in heart failure with reduced versus preserved ejection fraction. J Am Coll Cardiol. 2014;64:2281-93.

20. Seiler J, Stevenson WG. Atrial fibrillation in congestive heart failure. Cardiol Rev. 2010;18:38-50.

21. Thihalolipavan S, Morin DP. Atrial fibrillation and heart failure: update 2015. Prog Cardiovasc Dis. 2015;58:126-35.

22. Birnie DH, Tang AS. The problem of non-response to cardiac resynchronization therapy. Curr Opin Cardiol. 2006;21:20-6.

23. Mooyaart EA, Marsan NA, van Bommel RJ, Thijssen J, Borleffs CJ, Delgado V, et al. Comparison of long-term survival of men versus women with heart failure treated with cardiac resynchronization therapy. Am J Cardiol. 2011;108:63-8.

24. Sipahi I, Chou JC, Hyden M, Rowland DY, Simon DI, Fang JC. Effect of QRS morphology on clinical event reduction with cardiac resynchronization therapy: meta-analysis of randomized controlled trials. Am Heart J. 2012;163:260-7, e3.

25. Ypenburg C, van Bommel RJ, Delgado V, Mollema SA, Bleeker GB, Boersma E, et al. Optimal left ventricular lead position predicts reverse remodeling and survival after cardiac resynchronization therapy. J Am Coll Cardiol. 2008;52:1402-9. 